\title{
A FURI-PERA THEOREM IN HAUSDORFF TOPOLOGICAL SPACES FOR ACYCLIC MAPS
}

\author{
RAVI P. AGARWAL, JEWGENI H. DSHALALOW, and DONAL O'REGAN
}

Received 1 May 2004

We present new Furi-Pera theorems for acyclic maps between topological spaces.

2000 Mathematics Subject Classification: 47H10.

1. Introduction. In this paper, we present new Furi-Pera theorems $[6,7]$ for acyclic maps between Hausdorff topological spaces. The main result in our paper is based on a new Leray-Schauder alternative [1] for such maps which in turn is based on the notion of compactly null-homotopic.

We first recall some results and ideas from the literature. Let $X$ and $Z$ be subsets of Hausdorff topological spaces. We will consider maps $F: X \rightarrow K(Z)$; here $K(Z)$ denotes the family of nonempty compact subsets of $Z$. A nonempty topological space is said to be acyclic if all its reduced Čech homology groups over the rationals are trivial. Now $F: X \rightarrow K(Z)$ is acyclic if $F$ is upper semicontinuous with acyclic values. Suppose $X$ and $Z$ are topological spaces. Given a class $\mathscr{X}$ of maps, $\mathscr{X}(X, Z)$ denotes the set of maps $F: X \rightarrow 2^{Z}$ (nonempty subsets of $Z$ ) belonging to $\mathscr{X}$, and $\mathscr{X}_{c}$ the set of finite compositions of maps in $\mathscr{L}$. We let

$$
\mathscr{F}(\mathscr{X})=\{W: \operatorname{Fix} F \neq \varnothing \forall F \in \mathscr{L}(W, W)\},
$$

where Fix $F$ denotes the set of fixed points of $F$.

The class $U$ of maps is defined by the following properties:

(i) $U$ contains the class $\mathscr{b}$ of single-valued continuous functions;

(ii) each $F \in U_{c}$ is upper semicontinuous and compact valued;

(iii) $B^{n} \in \mathscr{F}\left(U_{c}\right)$ for all $n \in\{1,2, \ldots\}$; here $B^{n}=\left\{x \in \mathbb{R}^{n}:\|x\| \leq 1\right\}$.

Next we consider the class $u_{c}^{K}(X, Z)$ of maps $F: X \rightarrow 2^{Z}$ such that for each $F$ and each nonempty compact subset $K$ of $X$, there exists a map $G \in U_{c}(K, Z)$ such that $G(x) \subseteq F(x)$ for all $x \in K$. Notice the Kakutani and acyclic maps are examples of $u_{c}^{\kappa}$ maps (see $[3,4,8]$ for other examples).

By a space, we mean a Hausdorff topological space. Let $Q$ be a class of topological spaces. A space $Y$ is an extension space for $Q$ (written $Y \in \operatorname{ES}(Q)$ ) if for all $X \in Q$ and for all $K \subseteq X$ closed in $X$, any continuous function $f_{0}: K \rightarrow Y$ extends to a continuous function $f: X \rightarrow Y$.

For a subset $K$ of a topological space $X$, we denote by $\operatorname{Cov}_{X}(K)$ the set of all coverings of $K$ by open sets of $X$ (usually we write $\operatorname{Cov}(K)=\operatorname{Cov}_{X}(K)$ ). Let $Q$ be a class of 
topological spaces and $Y$ a subset of a Hausdorff topological space. Given two maps $F, G: X \rightarrow 2^{Y}$ and $\alpha \in \operatorname{Cov}(Y), F$ and $G$ are said to be $\alpha$-close if for any $x \in X$, there exists $U_{x} \in \alpha, y \in F(x) \cap U_{x}$, and $w \in G(x) \cap U_{x}$. A space $Y$ is an approximate extension space for $Q$ (written $Y \in \operatorname{AES}(Q)$ ) if for all $\alpha \in \operatorname{Cov}(Y)$, for all $X \in Q$, for all $K \subseteq X$ closed in $X$, and for any continuous function $f_{0}: K \rightarrow Y$, there exists a continuous function $f: X \rightarrow Y$ such that $\left.f\right|_{K}$ is $\alpha$-close to $f_{0}$.

Let $X$ be a uniform space. Then $X$ is Schauder admissible if for every compact subset $K$ of $X$ and every covering $\alpha \in \operatorname{Cov}_{X}(K)$, there exists a continuous function (called the Schauder projection) $\pi_{\alpha}: K \rightarrow X$ such that

(i) $\pi_{\alpha}$ and $i: K \hookrightarrow X$ are $\alpha$-close;

(ii) $\pi_{\alpha}(K)$ is contained in a subset $C \subseteq X$ with $C \in \operatorname{AES}$ (compact).

Let $X$ be a Hausdorff topological space and let $\alpha \in \operatorname{Cov}(X) . X$ is said to be Schauder admissible $\alpha$-dominated if there exist a Schauder admissible space $X_{\alpha}$ and two continuous functions $r_{\alpha}: X_{\alpha} \rightarrow X, s_{\alpha}: X \rightarrow X_{\alpha}$ such that $r_{\alpha} s_{\alpha}: X \rightarrow X$ and $i: X \rightarrow X$ are $\alpha$-close. $X$ is said to be almost Schauder admissible dominated if $X$ is Schauder admissible $\alpha$-dominated for each $\alpha \in \operatorname{Cov}(X)$. In [2], we established the following result.

Theorem 1.1. Let $X$ be a uniform space and let $X$ be almost Schauder admissible dominated. Also suppose $F \in U_{c}^{\kappa}(X, X)$ is a compact upper semicontinuous map with closed values. Then $F$ has a fixed point.

In our next definitions, $Y$ will be a completely regular topological space and $U$ an open subset of $Y$.

DEFINITION 1.2. $F \in \mathrm{AC}(\bar{U}, Y)$ if $F: \bar{U} \rightarrow K(Y)$ is an acyclic compact map; here $\bar{U}$ denotes the closure of $U$ in $Y$.

DeFinition 1.3. $F \in \mathrm{AC}_{\partial U}(\bar{U}, Y)$ if $F \in \mathrm{AC}(\bar{U}, Y)$ with $x \notin F(x)$ for $x \in \partial U$; here $\partial U$ denotes the boundary of $U$ in $Y$.

DEFINITION 1.4. $F \in \mathrm{AC}(Y, Y)$ if $F: Y \rightarrow K(Y)$ is an acyclic compact map.

DeFinition 1.5. If $F \in \mathrm{AC}(Y, Y)$ and $p \in Y$, then $F \cong\{p\}$ in $\mathrm{AC}(Y, Y)$ if there exists an acyclic compact map $R: Y \times[0,1] \rightarrow K(Y)$ with $R_{1}=F$ and $R_{0}=\{p\}$ (here $R_{t}(x)=$ $R(x, t))$.

The following three results were established in [1]. We note that Theorem 1.7 follows from Theorems 1.8, 1.1, and 1.6.

THEOREM 1.6. Let $Y$ be a metrizable ANR, $p \in Y$, and $F \in \operatorname{AC}(Y, Y)$ with $F \cong\{p\}$ in $\mathrm{AC}(Y, Y)$. Then $F$ has a fixed point.

THEOREM 1.7. Let $Y$ be a completely regular topological space, $U$ an open subset of $Y, u_{0} \in U$, and $F \in \mathrm{AC}_{\partial U}(\bar{U}, Y)$. Suppose there exists an acyclic compact map $H$ : $\bar{U} \times[0,1] \rightarrow K(Y)$ with $H_{1}=F, H_{0}=\left\{u_{0}\right\}$, and with $x \notin H_{t}(x)$ for $x \in \partial U$ and $t \in(0,1)$. In addition assume either of the following occurs:

(A) $Y$ is a uniform space and $Y$ is almost Schauder admissible dominated;

(B) $Y$ is a metrizable ANR.

Then $F$ has a fixed point. 
THEOREM 1.8. Let $Y$ be a completely regular topological space, $U$ an open subset of $Y, u_{0} \in U$, and $F \in \mathrm{AC}_{\partial U}(\bar{U}, Y)$. Suppose there exists an acyclic compact map $H$ : $\bar{U} \times[0,1] \rightarrow K(Y)$ with $H_{1}=F, H_{0}=\left\{u_{0}\right\}$, and with $x \notin H_{t}(x)$ for $x \in \partial U$ and $t \in(0,1)$. In addition, assume the following property holds:

$$
\begin{aligned}
& \text { for any } G \in \mathrm{AC}(Y, Y) \text { and any } p \in Y \text { with } G \cong\{p\} \\
& \text { in } \mathrm{AC}(Y, Y), G \text { has a fixed point in } Y .
\end{aligned}
$$

Then $F$ has a fixed point in $U$.

Let $Q$ be a subset of a Hausdorff topological space $X$. Then $Q$ is called a special retract of $X$ if there exists a continuous retraction $r: X \rightarrow Q$ with $r(x) \in \partial Q$ for $x \in X \backslash Q$.

EXAMPLE 1.9. Let $X$ be a Hilbert space and $Q$ a nonempty closed convex subset of $X$. Then $Q$ is a special retract of $X$ since we may take $r(\cdot)$ to be $P_{Q}(\cdot)$ which is the nearest point projection on $Q$.

EXAMPLE 1.10. Let $Q$ be a nonempty closed convex subset of a locally convex topological vector space $X$. Then we know from Dugundji's extension theorem that there exists a continuous retraction $r: X \rightarrow Q$. If int $Q=\varnothing$, then $\partial Q=Q$ so $r(x) \in \partial Q=Q$ if $x \in X$. Now suppose int $Q \neq \varnothing$. Without loss of generality, assume $0 \in \operatorname{int} Q$. Now we may take

$$
r(x)=\frac{x}{\max \{1, \mu(x)\}}, \quad x \in X
$$

where $\mu$ is the Minkowski functional on $Q$, that is, $\mu(x)=\inf \{\alpha>0: x \in \alpha Q\}$. Note, $r(x) \in \partial Q$ for $x \in X \backslash Q$, so $Q$ is a special retract of $X$.

2. Fixed point theory. In this section we present three Furi-Pera type theorems based on Theorems 1.1, 1.6-1.8.

THEOREM 2.1. Let $E=(E, d)$ be a metrizable space, $Q$ a closed subset of $E, u_{0} \in$ $Q$, and, $Q$ a special retract of $E$. Also assume $F \in \operatorname{AC}(Q, E)$ with $E$ almost admissible dominated. In addition, suppose the following condition is satisfied:

$$
\begin{aligned}
& \text { there exists an acyclic compact map } H: Q \times[0,1] \longrightarrow K(E) \\
& \text { with } H_{1}=F, H_{0}=\left\{u_{0}\right\} \text { such that if }\left\{\left(x_{j}, \lambda_{j}\right)\right\}_{j \in \mathbb{N}} \\
& \text { (here } \mathbb{N}=\{1,2, \ldots\}) \text { is a sequence in } \partial Q \times[0,1] \text { converging } \\
& \text { to }(x, \lambda) \text { with } x \in H(x, \lambda) \text { and } 0 \leq \lambda<1 \text {, then } \\
& \left\{H\left(x_{j}, \lambda_{j}\right)\right\} \subseteq Q \text { for } j \text { sufficiently large. }
\end{aligned}
$$

Then $F$ has a fixed point in $Q$.

Proof. Now since $Q$ is a special retract of $E$, there exists a continuous retraction $r: E \rightarrow Q$ with $r(z) \in \partial Q$ if $z \in E \backslash Q$. Consider

$$
B=\{x \in E: x \in \operatorname{Fr}(x)\}
$$


Clearly $F r: E \rightarrow K(E)$ is acyclic valued, upper semicontinuous, and compact. Thus $F r \in$ $\mathrm{AC}(E, E)$, so Theorem 1.1 guarantees that $B \neq \varnothing$. Also since $F r$ is upper semicontinuous we have that $B$ is closed. In fact, $B$ is compact since $F r$ is a compact map. It remains to show $B \cap Q \neq \varnothing$. To do this, we argue by contradiction. Suppose $B \cap Q=\varnothing$. Then since $B$ is compact and $Q$ is closed, there exists a $\delta>0$ with $\operatorname{dist}(B, Q)>\delta$. Choose $m \in \mathbb{N}=\{1,2, \ldots\}$ with $1<\delta m$. Let

$$
U_{i}=\left\{x \in E: d(x, Q)<\frac{1}{i}\right\} \quad \text { for } i \in\{m, m+1, \ldots\} .
$$

Fix $i \in\{m, m+1, \ldots\}$. Now since $\operatorname{dist}(B, Q)>\delta$, then $B \cap \overline{U_{i}}=\varnothing$. Notice also that $U_{i}$ is open, $u_{0} \in U_{i}$, and $F r: \overline{U_{i}} \rightarrow K(E)$ is an upper semicontinuous, acyclic valued, and compact map (i.e., $F r \in \mathrm{AC}\left(\overline{U_{i}}, E\right)$ ). Let $H: Q \times[0,1] \rightarrow K(E)$ be an acyclic compact map with $H_{1}=F, H_{0}=\left\{u_{0}\right\}$ as described in (2.1). Now let $R: \overline{U_{i}} \times[0,1] \rightarrow K(E)$ be given by $R(x, t)=H(r(x), t)$. Clearly $R: \overline{U_{i}} \times[0,1] \rightarrow K(E)$ is an acyclic compact map with $R_{1}=F r$ and $R_{0}=\left\{u_{0}\right\}$. Now $B \cap \overline{U_{i}}=\varnothing$, together with Theorem 1.7, guarantees that there exists

$$
\left(y_{i}, \lambda_{i}\right) \in \partial U_{i} \times(0,1) \text { with } y_{i} \in H\left(r\left(y_{i}\right), \lambda_{i}\right) .
$$

We can do this for each $i \in\{m, m+1, \ldots\}$. Consequently,

$$
\left\{H\left(r\left(y_{j}\right), \lambda_{j}\right)\right\} \nsubseteq Q \quad \text { for each } j \in\{m, m+1, \ldots\} \text {. }
$$

We now look at

$$
D=\left\{x \in E: x \in R_{\lambda}(r(x)) \text { for some } \lambda \in[0,1]\right\} .
$$

Now $D \neq \varnothing$ is closed and in fact compact (so sequentially compact). This together with

$$
d\left(y_{j}, Q\right)=\frac{1}{j}, \quad\left|\lambda_{j}\right| \leq 1 \quad \text { for } j \in\{m, m+1, \ldots\}
$$

implies that we may assume without loss of generality that

$$
\lambda_{j} \longrightarrow \lambda^{\star} \in[0,1], \quad y_{j} \longrightarrow y^{\star} \in \partial Q
$$

In addition $y_{j} \in H\left(r\left(y_{j}\right), \lambda_{j}\right)$ with $R$ upper semicontinuous (so closed, [5, page 465]) guarantees that $y^{\star} \in H\left(r\left(y^{\star}\right), \lambda^{\star}\right)$. Now if $\lambda^{\star}=1$, then $y^{\star} \in H\left(r\left(y^{\star}\right), 1\right)=\operatorname{Fr}\left(y^{\star}\right)$ which contradicts $B \cap Q=\varnothing$. Thus $0 \leq \lambda^{\star}<1$. But then (2.1) with $x_{j}=r\left(y_{j}\right) \in \partial Q$ (note that $Q$ is a special retract of $E$ ) and $x=y^{\star}=r\left(y^{\star}\right)$ implies $\left\{H\left(r\left(y_{j}\right), \lambda_{j}\right)\right\} \subseteq Q$ for $j$ sufficiently large. This contradicts (2.5). Thus $B \cap Q \neq \varnothing$, so there exists $x \in Q$ with $x \in \operatorname{Fr}(x)=F(x)$.

REMARK 2.2. We can remove the assumption that $Q$ is a special retract of $E$ provided we assume that

$$
\text { there exists a retraction } r: E \rightarrow Q \text {, }
$$


and (2.1) is replaced by the following:

$$
\begin{aligned}
& \text { there exists an acyclic compact map } H: Q \times[0,1] \longrightarrow K(E) \\
& \text { with } H_{1}=F, H_{0}=\left\{u_{0}\right\} \text { such that if }\left\{\left(x_{j}, \lambda_{j}\right)\right\}_{j \in \mathbb{N}} \\
& \text { (here } \mathbb{N}=\{1,2, \ldots\}) \text { is a sequence in } Q \times[0,1] \text { converging } \\
& \text { to }(x, \lambda) \text { with } x \in H(x, \lambda) \text { and } 0 \leq \lambda<1 \text {, then } \\
& \left\{H\left(x_{j}, \lambda_{j}\right)\right\} \subseteq Q \text { for } j \text { sufficiently large. }
\end{aligned}
$$

THEOREM 2.3. Let $E=(E, d)$ be a metrizable space, $Q$ a closed subset of $E, u_{0} \in Q$, and $Q$ a special retract of $E$. Also assume $F \in \mathrm{AC}(Q, E)$ with $E$ an ANR. In addition, assume (2.1) is satisfied and that the following condition holds:

$$
\begin{aligned}
& \text { for any } G \in \mathrm{AC}(E, E) \text { and any } p \in E \text {, there exists } \\
& \text { an acyclic compact map } \Phi: E \times[0,1] \longrightarrow K(E) \text { with } \\
& \Phi_{1}=G \text { and } \Phi_{0}=\{p\}\left(\text { here } \Phi_{t}(x)=\Phi(t, x)\right) \text {. }
\end{aligned}
$$

Then $F$ has a fixed point in $Q$.

Proof. Let $r$ and $B$ be as in the proof of Theorem 2.1. Notice $F r \in \mathrm{AC}(E, E)$. Fix $p \in$ $E$. Now (2.11) guarantees that there exists an acyclic compact map $\Psi: E \times[0,1] \rightarrow K(E)$ with $\Psi_{1}=F r$ and $\Psi_{0}=\{p\}$. This together with Theorem 1.6 guarantees that $B \neq \varnothing$. Essentially the same reasoning as in Theorem 2.1 establishes the result.

REMARK 2.4. In Theorem 2.3, we can replace " $Q$ is a special retract of $E$ " provided we assume (2.9) and replace (2.1) with (2.10).

REMARK 2.5. From the proof of Theorem 2.3, we can see immediately that (2.11) could be replaced by the following:

$$
\begin{aligned}
& \text { there exist } p \in E \text { and an acyclic compact map } \\
& \Phi: E \times[0,1] \longrightarrow K(E) \text { with } \Phi_{1}=F r \text { and } \Phi_{0}=\{p\} .
\end{aligned}
$$

Our next result is a generalization of Theorem 2.3.

TheOREM 2.6. Let $E=(E, d)$ be a metrizable space, $Q$ a closed subset of $E, u_{0} \in Q$, and $Q$ a special retract of $E$. Also assume $F \in \mathrm{AC}(Q, E)$ and that (2.1) and (2.11) are satisfied. In addition, suppose the following condition holds:

$$
\begin{aligned}
& E \text { is such that for any } G \in \mathrm{AC}(E, E) \text { and any } \\
& p \in E \text { with } G \cong\{p\} \text { in } \mathrm{AC}(E, E), \\
& G \text { has a fixed point. }
\end{aligned}
$$

Then $F$ has a fixed point in $Q$.

Proof. Let $r$ and $B$ be as in the proof of Theorem 2.1. The argument in Theorem 2.3 guarantees that $B \neq \varnothing$. Also of course $B$ is closed and compact. Suppose $B \cap Q=\varnothing$. Then there exists a $\delta>0$ with $\operatorname{dist}(B, Q)>\delta$. Choose $m \in \mathbb{N}=\{1,2, \ldots\}$ with $1<$ $\delta m$ and let $U_{i}(i \in\{m, m+1, \ldots\})$ be as in Theorem 2.1. Fix $i \in\{m, m+1, \ldots\}$. Note $B \cap \overline{U_{i}}=\varnothing$ and $F r \in \mathrm{AC}\left(\overline{U_{i}}, E\right)$. Let $H: Q \times[0,1] \rightarrow K(E)$ be an acyclic compact map 
with $H_{1}=F, H_{0}=\left\{u_{0}\right\}$ as described in (2.1) and let $R: \overline{U_{i}} \times[0,1] \rightarrow K(E)$ be given by $R(x, t)=H(r(x), t)$. Clearly $R: \overline{U_{i}} \times[0,1] \rightarrow K(E)$ is an acyclic compact map with $R_{1}=$ Fr and $R_{0}=\left\{u_{0}\right\}$. Now $B \cap \overline{U_{i}}=\varnothing,(2.13)$, and Theorem 1.8 guarantee that there exists $\left(y_{i}, \lambda_{i}\right) \in \partial U_{i} \times(0,1)$ with $y_{i} \in H\left(r\left(y_{i}\right), \lambda_{i}\right)$. We can do this for each $i \in\{m, m+1, \ldots\}$. Consequently $\left\{H\left(r\left(y_{j}\right), \lambda_{j}\right)\right\} \nsubseteq Q$ for each $j \in\{m, m+1, \ldots\}$. Essentially the same reasoning as in Theorem 2.1 from (2.5) onwards establishes the result.

REMARK 2.7. In Theorem 2.6, we can replace " $Q$ is a special retract of $E$ " provided we assume (2.9) and replace (2.1) with (2.10).

REMARK 2.8. In Theorem 2.6, note (2.11) could be replaced by (2.12).

\section{REFERENCES}

[1] R. P. Agarwal, J. H. Dshalalow, and D. O'Regan, Generalized Leray-Schauder principles in Hausdorff topological spaces for acyclic maps, to appear in Math. Sci. Res. J.

[2] R. P. Agarwal, J. K. Kim, and D. O'Regan, Fixed point theory for composite maps on almost dominating extension spaces, to appear.

[3] R. P. Agarwal and D. O'Regan, Homotopy and Leray-Schauder principles for multi maps, Nonlinear Anal. Forum 7 (2002), no. 1, 103-111.

[4] R. P. Agarwal, D. O'Regan, and S. Park, Fixed point theory for multimaps in extension type spaces, J. Korean Math. Soc. 39 (2002), no. 4, 579-591.

[5] C. D. Aliprantis and K. C. Border, Infinite-Dimensional Analysis, Studies in Economic Theory, vol. 4, Springer-Verlag, Berlin, 1994.

[6] M. Furi and A. Vignoli, An extension of the notion of zero-epi maps to the context of topological spaces, Z. Anal. Anwendungen 21 (2002), no. 2, 485-493.

[7] D. O'Regan, Fixed point theory for closed multifunctions, Arch. Math. (Brno) 34 (1998), no. 1, 191-197.

[8] _ Fixed point theory on extension-type spaces and essential maps on topological spaces, Fixed Point Theory Appl. 2004 (2004), no. 1, 13-20.

Ravi P. Agarwal: Department of Mathematical Sciences, Florida Institute of Technology, Melbourne, FL 32901-6975, USA

E-mail address: agarwa1@fit.edu

Jewgeni H. Dshalalow: Department of Mathematical Sciences, Florida Institute of Technology, Melbourne, FL 32901-6975, USA

E-mail address: eugene@fit.edu

Donal O'Regan: Department of Mathematics, National University of Ireland, Galway, Ireland

E-mail address: dona7 . oregan@nuiga 7way. i e 


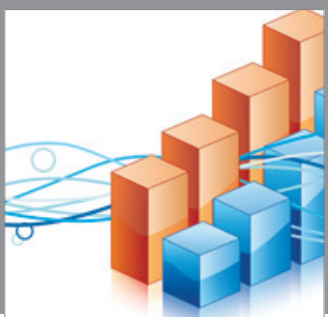

Advances in

Operations Research

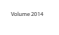

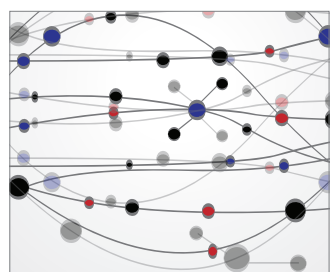

\section{The Scientific} World Journal
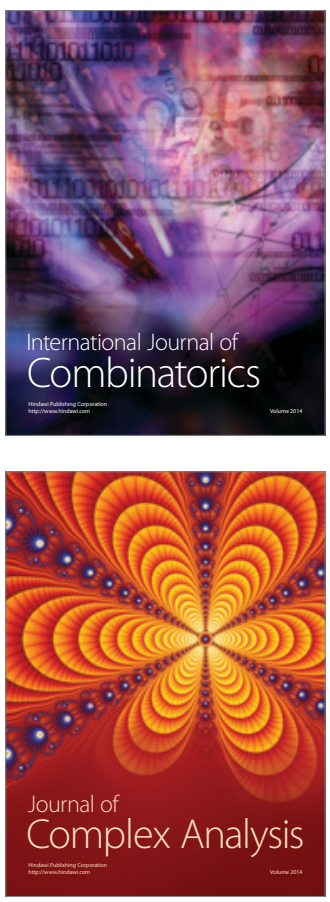

International Journal of

Mathematics and

Mathematical

Sciences
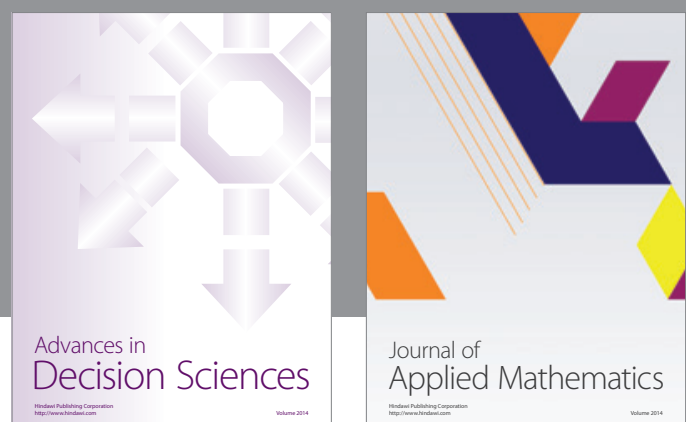

Journal of

Applied Mathematics
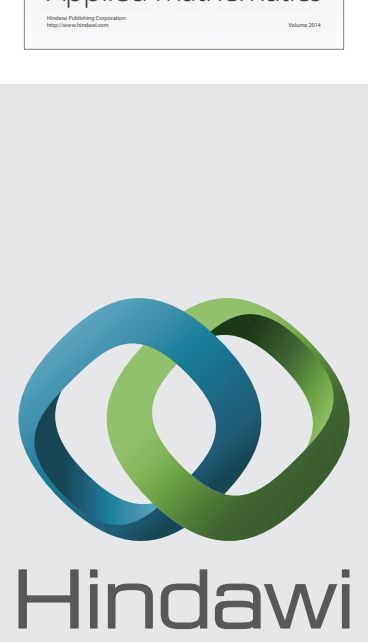

Submit your manuscripts at http://www.hindawi.com
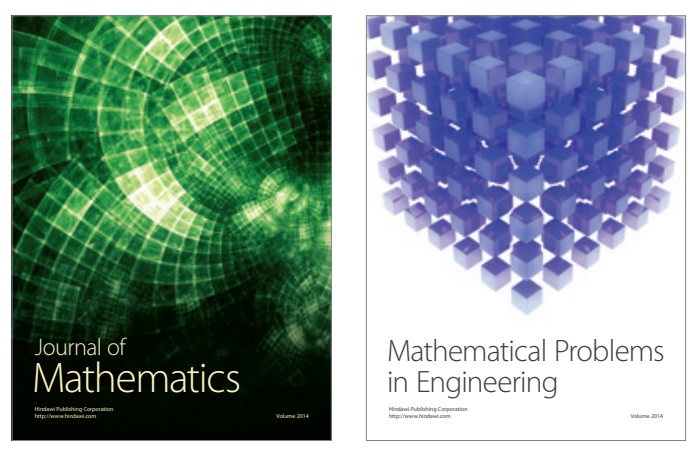

Mathematical Problems in Engineering
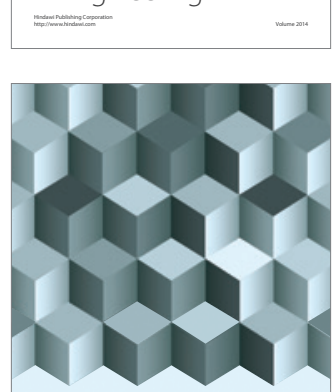

Journal of

Function Spaces
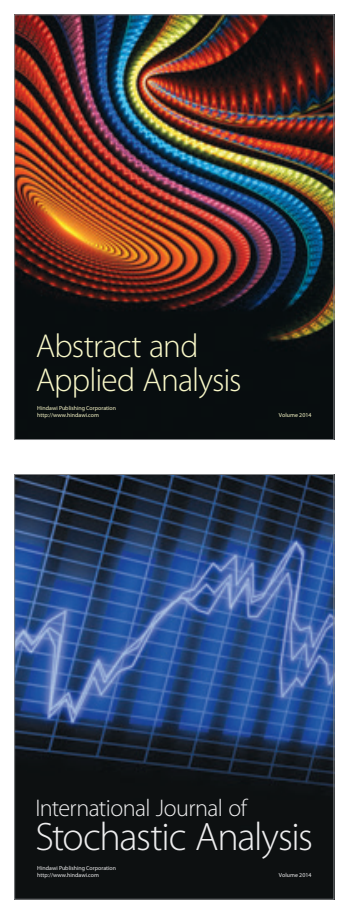

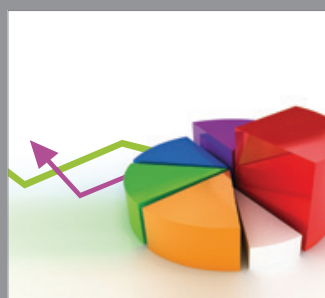

ournal of

Probability and Statistics

Promensencen
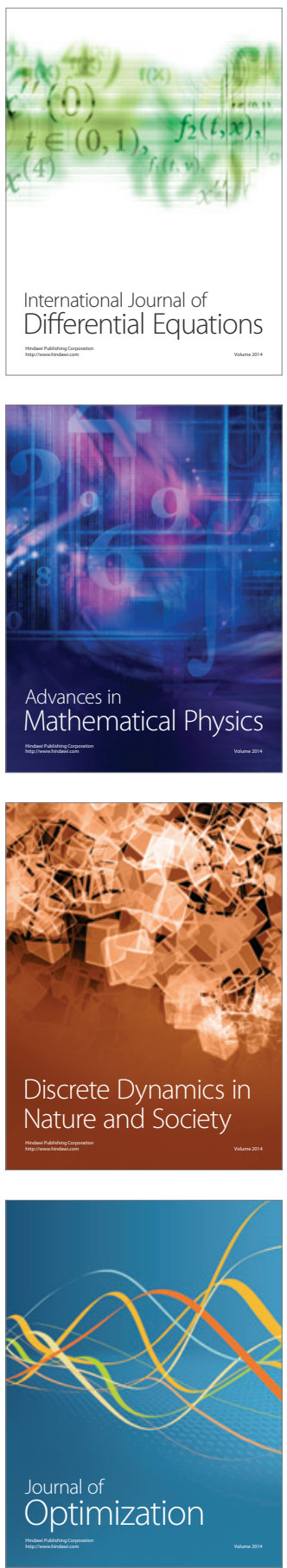